\title{
A comparison between the spectral and long term timing properties of GX 3+1, GX 5-1 and GX 13+1
}

\author{
Petri SAVOLAINEN* \\ Aalto University Metsähovi Radio Observatory, Metsähovintie 114, FIN-02540 Kylmälä, Finland \\ E-mail: petri.savolainenetkk.fi
}

\section{Diana Carina HANNIKAINEN}

Aalto University Metsähovi Radio Observatory / Finnish Centre for Astronomy with ESO,

Finland

E-mail: diana@kurp.hut.fi

\section{Ada PAIZIS}

INAF - Instituto di Astrofisica Spaziale e Fisica Cosmica, Sezione di Milano, Milano, Italy

E-mail: ada@iasf-milano.inaf.it

\section{Ruben FARINELLI}

Dipartimento di Fisica, Universitá di Ferrara, Ferrara, Italy

E-mail: farinelli@fe.infn.it

\section{Erik KUULKERS}

ISOC, ESA/ESAC, Madrid, Spain

E-mail: Erik.Kuulkersesciops.esa.int

\section{Osmi VILHU}

Observatory, University of Helsinki, Finland

E-mail: osmi.vilhu@helsinki.fi

Using GX 3+1 as an example of a bright Atoll, GX 5-1 as an example of a Z source, and GX $13+1$ as a peculiar hybrid, we take a look at the spectral and long-term time evolution differences in these three neutron star Low-Mass X-ray Binaries representing different subtypes. We utilize a recently developed spectral model based on mixed thermal and bulk motion Comptonization of soft seed photons from the accretion disc and the neutron star surface. The hard X-ray tails exhibited by GX 5-1 and GX 13+1 are attributed to efficient bulk motion Comptonization, which is not present in GX $3+1$ due to too low or too high a local accretion rate. Accreting well below the Eddington limit also enables GX $3+1$ to exhibit amplitude modulation presumably due to changes in the accretion output from the companion star, visible in the RXTE/ASM light curve on a time scale of $\sim 6$ years.

8th INTEGRAL Workshop, "The Restless Gamma-ray Universe”,

September 27-30, 2010

Dublin, Ireland

\footnotetext{
* Speaker.
} 


\section{Introduction}

GX $3+1$ is a persistently bright Atoll-type neutron star Low-Mass X-ray Binary (NS LMXB) that has long been known to show flux variations of a factor of $\sim 2-3$ on time scales of several years (Makishima et al., 1983; Asai et al., 1993; Kotze \& Charles, 2010). Kuulkers \& van der Klis (2000) found one X-ray burst that shows evidence for radius expansion of the neutron star photosphere during a thermonuclear runaway, suggesting temporary super-Eddington emission. The binary companion and orbital period remain undetected.

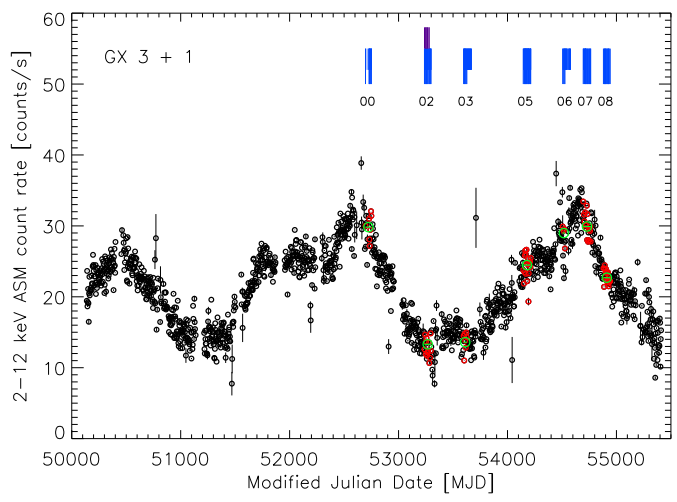

Figure 1: The RXTE/ASM light curve of GX 3+1 from 1996 February to 2010 August. The points corresponding to near-simultaneous INTEGRAL observations (long blue lines) are shown in red, and the green circles represent the weighted averages (per INTEGRAL observing interval) of the red data points. The INTEGRAL observations span from 2003 March to 2009 April. Interval 02 also has near-simultaneous observations by RXTE (purple lines).

GX 13+1 was classified as an Atoll source by Hasinger \& van der Klis (1989), as its X-ray colour-colour diagram and power spectrum placed it in the banana state. This is at odds with other studies grouping it together with the more luminous NS LMXBs (Parsignault \& Grindlay, 1978; Ponman, 1982; Schulz et al., 1989), and, given the theory that the dichotomy in NS LMXBs is due to their companions (van Paradijs \& Lewin, 1986), observations suggesting that the secondary is a K giant (Garcia et al., 1992; Bandyopadhyay et al., 1999), including those supporting a $\sim 25 \mathrm{~d}$ orbital period (Bandyopadhyay et al., 2002; Corbet, 2003). GX 13+1 also displays radio emission (Grindlay \& Seaquist, 1986), a $\sim 60 \mathrm{~Hz}$ Quasi-periodic oscillation (QPO) (Homan et al., 1998), and a transient hard tail (Paizis et al., 2006), which are otherwise associated with Z sources, as well as iron $\mathrm{K}$ absorption features similar to those in apparently superluminal black hole X-ray binaries (Ueda et al., 2001; Sidoli et al., 2002). In any case, type I X-ray bursts indicate it to be a neutron star source (Fleischman, 1985; Matsuba et al., 1995).

The Z source GX 5-1 is the second brightest persistent X-ray source after Sco X-1. QPOs have been detected in the X-ray light curves at various frequencies (e.g. van der Klis et al. 1985; Wijnands et al. 1999), and extensively studied since. Naylor et al. (1991) identified several candidate infrared counterparts, one of which was confirmed by Bandyopadhyay et al. (2003), but the type of the mass donor and the orbital period are yet to be established. GX 5-1 has a hard tail above 

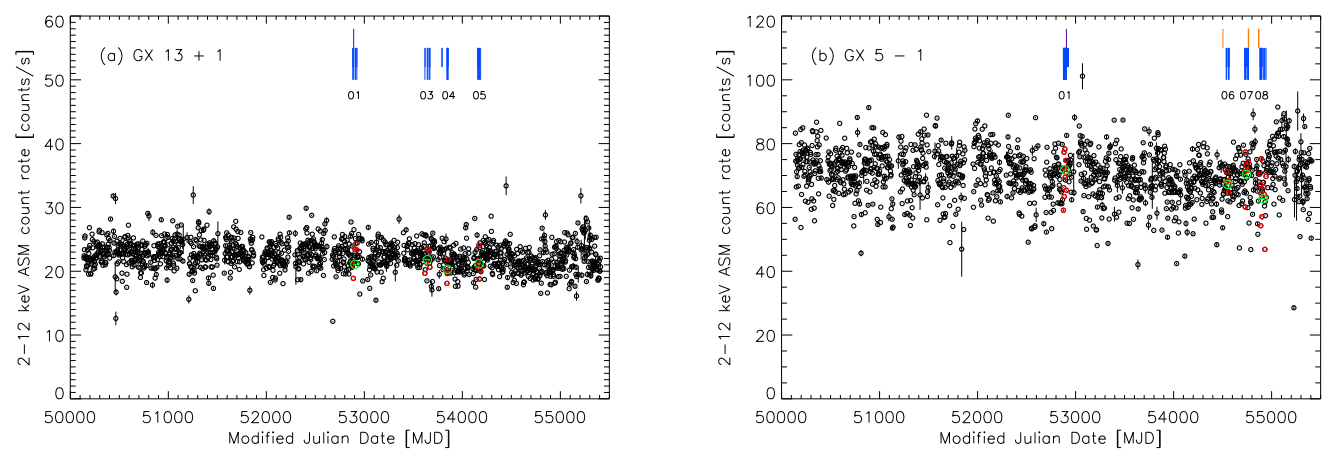

Figure 2: (a) The RXTE/ASM light curve of GX 13+1 from 1996 February to 2010 August. (b) The RXTE/ASM light curve of GX 5-1 from 1996 February to 2010 August. Note that the y-scale here is twice that of Figs. 1 and 2(a).

$20 \mathrm{keV}$ (Paizis et al., 2005), the study of which has been complicated by contamination from the nearby (40') black hole candidate GRS 1758-258 (Asai et al., 1994).

\section{Observations, data reduction and modeling}

Our INTEGRAL data sample was selected from Science Windows (ScWs) with offsets $<4^{\circ}$ and more than $1000 \mathrm{~s}$ of JEM-X1 or JEM-X2 and IBIS/ISGRI data. The sources have been observed by INTEGRAL recurrently within intervals of $\sim 70$ days twice a year. For each source, we picked a few of these intervals that had relatively good coverage (Figs. $1 \& 2$ ). Most of the data is from GX $3+1$, as it has the best INTEGRAL coverage and the most complex long-term RXTE All-Sky Monitor (ASM) light curve (Fig. 1). The data were reduced with the Off-line Science Analysis (OSA) version 8 software. We have also analyzed RXTE PCA and HEXTE spectra which were available for our INTEGRAL observing intervals.

For modeling the spectra with XSPEC 12, we used the COMPTB model recently introduced by Farinelli et al. (2008), and employed to study BeppoSAX spectra of Cyg X-2 in Farinelli et al. (2009), and several NS LMXBs in Mainardi et al. (2010). СОМРTB combines the thermal and bulk motion Comptonization of a seed photon spectrum to create a unified physical model that seeks to explain the spectral evolution of NS LMXBs. In this scenario, thermal Comptonization operates on soft accretion disc blackbody seed photons in the hot plasma of the outer Transition Layer (TL) between the accretion disc and the NS, while photons from the NS surface and inner part of the TL may experience both thermal and bulk motion Comptonization in a free-fall region which may form in the inner TL, depending on the accretion rate and resulting radiation pressure (Fig. 3 shows a schematic of the model).

The INTEGRAL and RXTE spectra were fitted with three model variations of increasing complexity: (1) a single СОМРТВ with thermal Comptonization only (Fig. 4); (2) two СОМРТВ components, one of which thermally Comptonized and the other reduced to a blackbody (Fig. 5(a)); (3) two СОмРтв components, one of which only thermally and the other thermally as well as bulk motion Comptonized (Fig. 5(b)). The absorbing hydrogen columns (model tbabs) were set to values from literature. 


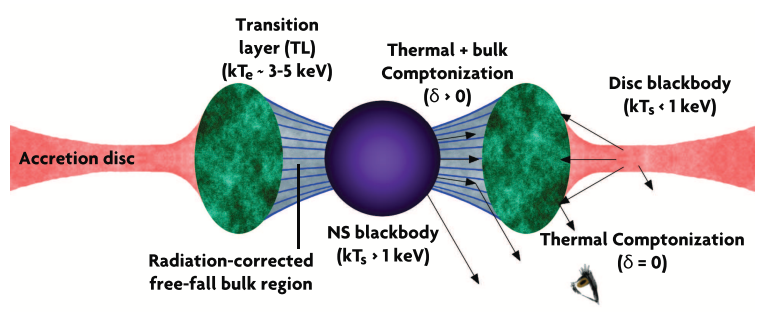

Figure 3: Schematic of the accretion/emission model used in this work.

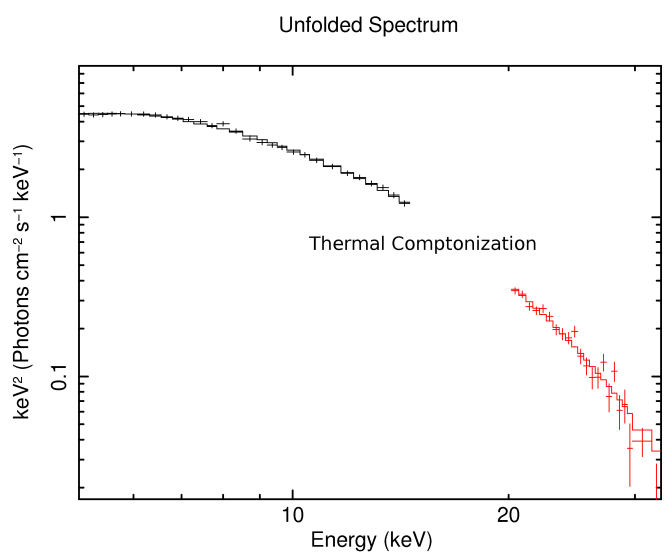

Figure 4: INTEGRAL JEM-X1 (black) and IBIS/ISGRI (red) spectrum of the Atoll source GX 3+1 from interval 07 (see Fig. 1) with the best-fit model of thermal Comptonization of $\mathrm{k} T_{\mathrm{s}} \lesssim 1 \mathrm{keV}$ blackbody photons from a $\mathrm{k} T_{\mathrm{e}} \sim 3-4 \mathrm{keV}$ electron plasma. Three of the seven interval-averaged GX 3+1 spectra were best fit with this single-component version. The other four spectra favored a second component (see Fig. 5).

\section{Results and discussion}

In terms of the accretion scenario used in this study, the hard X-ray tails exhibited by GX 5-1 and GX 13+1 (as well as other Z sources) can be explained by efficient bulk motion Comptonization in a freefall region near the NS surface, i.e. the inner TL. The hard emission is observed while the sources are in the Horizontal Branch (e.g. Paizis et al. 2005), corresponding to a certain range of local accretion rates. When the rate is increased, radiation pressure stops the bulk motion, suppressing the hard emission in the Normal and Flaring branches. This may be the case permanently in the bright Atoll sources such as GX 3+1, or possibly the local accretion rate is too low for bulk motion Comptonization to produce observable hard emission.

Changes in the matter transfer rate from the companion due to a Solar-like magnetic cycle have been suggested as the origin of the long-term modulation in GX $3+1$ and other persistent Atoll sources (Kotze \& Charles, 2010). Z sources could be less affected due to the output from the donor exceeding the Eddington limit for accretion onto the NS; the local accretion/emission rate remains near the Eddington limit and the surplus matter gets ejected. The cycle of the evolved companions could also be too long or too weak to be observed. 

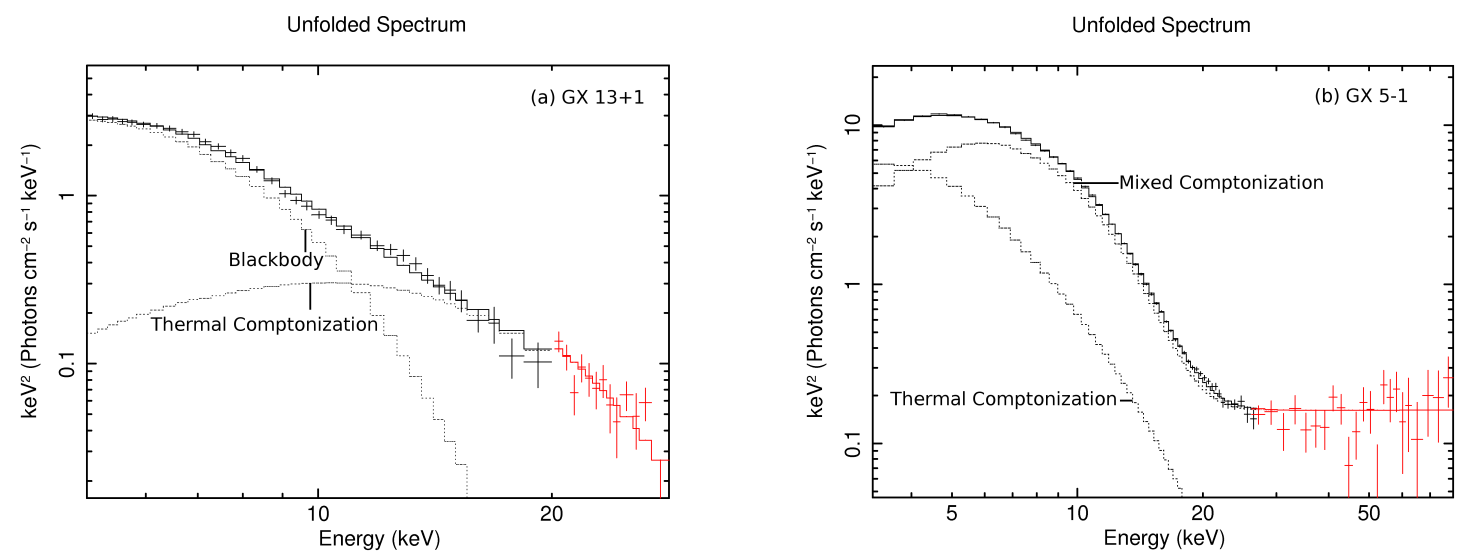

Figure 5: (a) INTEGRAL JEM-X1 (black) and IBIS/ISGRI (red) spectrum of the Atoll source GX 13+1 from interval 01 (see Fig. 2(a)) with the best-fit thermal Comptonization + blackbody model. This model version was favored by three out of four of the GX $13+1$ spectra: thermal Comptonization of soft photons dominates, while a harder $(>1 \mathrm{keV})$ blackbody-like component is also discernible. The spectrum from interval 04 was harder, supporting bulk motion Comptonization (the model of Fig. 5(b)). (b) RXTE PCA (black) + HEXTE (red) spectrum for the Z source GX 5-1 from interval 01 (see Fig. 2(b)) with the best-fit thermal Comptonization + mixed (thermal and bulk) Comptonization model. The spectrum from this interval showed an unambiguous hard tail which required bulk motion Comptonization to fit it, while the other three GX 5-1 intervals could be adequately fit also with the simpler model versions.

\section{Future work}

We have studied the few available Swift spectra of GX 5-1, and are presently looking at Chandra grating spectra, as a means of establishing the low-energy parameters such as the seed photon temperatures. This will allow the Comptonization parameters to be better constrained.

\section{Acknowledgements}

PS acknowledges support from the Magnus Ehrnrooth foundation, grants 2009t19 and TA2010n9.

\section{References}

Asai K., et al., 1993, PASJ, 45, 801

Asai K., Dotani T., Mitsuda K., Nagase F., Kamado Y., Kuulkers E., Breedon L. M., 1994, PASJ, 46,479

Bandyopadhyay R. M., Shahbaz T., Charles P. A., 2003, MNRAS, 340, L13

Bandyopadhyay R. M., Shahbaz T., Charles P. A., Naylor T., 1999, MNRAS, 306, 417

Bandyopadhyay R. M., Charles P. A., Shahbaz T., Wagner R. M., 2002, ApJ, 570, 793

Corbet R. H. D., 2003, ApJ, 595, 1086 
Farinelli R., Paizis A., Landi R., Titarchuk L., 2009, A\&A, 498, 509

Farinelli R., Titarchuk L., Paizis A., Frontera F., 2008, ApJ, 680, 602

Fleischman J. R., 1985, A\&A, 153, 106

Garcia M. R., Grindlay J. E., Bailyn C. D., Pipher J. L., Shure M. A., Woodward C. E., 1992, AJ, 103,1325

Grindlay J. E., Seaquist E. R., 1986, ApJ, 310, 172

Hasinger G., van der Klis M., 1989, A\&A, 225, 79

Homan J., van der Klis M., Wijnands R., Vaughan B., Kuulkers E., 1998, ApJ, 499, L41

Kotze M. M., Charles P. A., 2010, MNRAS, 402, L16

Kuulkers E., van der Klis M., 2000, A\&A, 356, L45

Mainardi L. I., Paizis A., Farinelli R., Kuulkers E., Rodriguez J., Hannikainen D., Savolainen P., Piraino S., Bazzano A., Santangelo A., 2010, A\&A, 512, id.A57

Makishima K., et al., 1983, ApJ, 267, 310

Matsuba E., Dotani T., Mitsuda K., Asai K., Lewin W. H. G., van Paradijs J., van der Klis M., 1995, PASJ, 47, 575

Naylor T., Charles P. A., Longmore A. J., 1991, MNRAS, 252, 203

Paizis A., et al., 2006, A\&A, 459, 187

Paizis A., Ebisawa K., Tikkanen T., Rodriguez J., Chenevez J., Kuulkers E., Vilhu O., Courvoisier T. J.-L., 2005, A\&A, 443, 599

Parsignault D. R., Grindlay J. E., 1978, ApJ, 225, 970

Ponman T., 1982, MNRAS, 201, 769

Schulz N. S., Hasinger G., Truemper J., 1989, A\&A, 225, 48

Sidoli L., Parmar A. N., Oosterbroek T., Lumb D., 2002, A\&A, 385, 940

Ueda Y., Asai K., Yamaoka K., Dotani T., Inoue H., 2001, ApJ, 556, L87

van der Klis M., Jansen F., van Paradijs J., Lewin W. H. G., van den Heuvel E. P. J., Trümper J. E., Sztjano M., 1985, Nat, 316, 225

van Paradijs J., Lewin W. H. G., 1986, in The Evolution of Galactic X-ray Binaries, ed. Trumper J., Lewin W. H. G., Brinkman W., Reidel, Boston, 187

Wijnands R., Meñdez M., van der Klis M., Psaltis D., Kuulkers E., Lamb F. K., 1999, ApJ, 504, L35 